\title{
APPROXIMATION OF BESOV VECTORS BY PALEY-WIENER VECTORS IN HILBERT SPACES
}

\author{
Isaac Z. Pesenson 1 \\ Meyer Z. Pesenson 2
}

\begin{abstract}
We develop an approximation theory in Hilbert spaces that generalizes the classical theory of approximation by entire functions of exponential type. The results advance harmonic analysis on manifolds and graphs, thus facilitating data representation, compression, denoising and visualization. These tasks are of great importance to machine learning, complex data analysis and computer vision.
\end{abstract}

\section{INTRODUCTION}

One of the main themes in Analysis is correlation between frequency content of a function and its smoothness. In the classical approach the frequency is understood in terms of the Fourier transform (or Fourier series) and smoothness is described in terms of the Sobolev or Lipshitz and Besov norms. For these notions it is well understood [1, 10] that there exists a perfect balance between the rate of approximation by bandlimited functions ( trigonometric polynomials) and smoothness described by Besov norms. For more recent results of approximations by entire functions of exponential type we refer to [5]- 7 .

The classical concepts and result were generalized to Riemannian manifolds, graphs, unitary representations of Lie groups and integral transforms in our work [11]- 21], 8 .

The goal of the present article is to develop a form of a Harmonic Analysis which holds true in general Hilbert spaces. In Introduction we formulate main results obtained in the paper. The exact definitions of all notions are given in the text.

We start with a self-adjoint positive definite operator $L$ in a Hilbert space $\mathcal{H}$ and consider its positive root $D=L^{1 / 2}$. For the operator $D$ one can introduce notion of the Spectral Transform $\mathcal{F}_{D}$ which is an isomorphism between $\mathcal{H}$ and a direct integral of Hilbert spaces over $\mathbb{R}$.

A Paley-Wiener space $P W_{\omega}(D), \omega>0$, is introduced as the set of all $f \in \mathcal{H}$ whose image $\mathcal{F}_{D} f$ has support in $[0, \omega]$. In the case when $\mathcal{H}=L_{2}(\mathbb{R})^{d}$ and $D$ is

1991 Mathematics Subject Classification. 43A80; Secondary 41A15, 41A17.

Key words and phrases. Self-adjoint operator, Paley-Wiener vectors, $K$-functor, Schrodinger goup of operators, Besov norms.

1 Department of Mathematics, Temple University, Philadelphia, PA 19122; pesenson@temple.edu. The author was supported in part by the National Geospatial-Intelligence Agency University Research Initiative (NURI), grant HM1582-08-1-0019.

${ }^{2}$ CMS Department, California Institute of Technology, MC 305-16, Pasadena, CA 91125; mzp@cms.caltech.edu. The author was supported in part by the National Geospatial-Intelligence Agency University Research Initiative (NURI), grant HM1582-08-1-0019 and by AFOSR, MURI, Award FA9550-09-1-0643 
a positive square root from the Laplace operator our definition produces regular Paley-Wiener spaces of spherical exponential type.

The domain $\mathcal{D}_{s}, s \in \mathbb{R}$, of the operator $D^{s}, s \in \mathbb{R}$, plays the role of the Sobolev space and we introduce Besov spaces $\mathbf{B}_{2, q}^{\alpha}=\mathbf{B}_{2, q}^{\alpha}(D), \alpha>0,1 \leq q \leq \infty$, by using Peetre's interpolation $K$-functor [2], 4, 8], [9, [23].

$$
\mathbf{B}_{2, q}^{\alpha}(D)=\left(\mathcal{H}, \mathcal{D}_{r / 2}\right)_{\alpha / r, q}^{K},
$$

where $r$ can be any natural such that $0<\alpha<r, 1 \leq q<\infty$, or $0 \leq \alpha \leq r, q=\infty$. It is crucial for us that Besov norms can be described in terms of a modulus of continuity constructed in terms of the Schrodinger group $e^{i t D^{2}}$, wave semigroup $e^{i t D}$, or the heat semigroup $e^{-t D^{2}}$. In what follows the notation $\|\cdot\|$ bellow means $\|\cdot\|_{\mathcal{H}}$. We introduce a notion of best approximation

$$
\mathcal{E}(f, \omega)=\inf _{g \in P W_{\omega}(D)}\|f-g\|, f \in \mathcal{H} .
$$

We also consider the following family of functionals which describe a rate of decay of the Spectral transform $\mathcal{F}_{D}$

$$
\mathcal{R}(f, \omega)=\left(\int_{\omega}^{\infty}\left\|\mathcal{F}_{D}(f)(\lambda)\right\|_{X(\lambda)}^{2} d m(\lambda)\right)^{1 / 2}, \omega>0 .
$$

The Plancherel Theorem for $\mathcal{F}_{D}$ implies that every such functional is exactly the best approximation of $f$ by Paley-Wiener functions from $P W_{\omega}(D)$ :

$$
\mathcal{R}(f, \omega)=\mathcal{E}(f, \omega)=\inf _{g \in P W_{\omega}(D)}\|f-g\| .
$$

Our main results are the following.

Theorem 1.1. The norm of the Besov space $\mathbf{B}_{2, q}^{\alpha}(D), \alpha>0,1 \leq q \leq \infty$ is equivalent to the following norms

and

$$
\begin{gathered}
\|f\|+\left(\int_{0}^{\infty}\left(s^{\alpha} \mathcal{E}(f, s)\right)^{q} \frac{d s}{s}\right)^{1 / q} \\
\|f\|+\left(\sum_{k=0}^{\infty}\left(a^{k \alpha} \mathcal{E}\left(f, a^{k}\right)\right)^{q}\right)^{1 / q}, a>1 . \\
\|f\|+\left(\int_{0}^{\infty}\left(s^{\alpha} \mathcal{R}(f, s)\right)^{q} \frac{d s}{s}\right)^{1 / q}
\end{gathered}
$$

$$
\|f\|+\left(\sum_{k=0}^{\infty}\left(a^{k \alpha} \mathcal{R}\left(f, a^{k}\right)\right)^{q}\right)^{1 / q}, a>1 .
$$

Theorem 1.2. A vector $f \in \mathcal{H}$ belongs to $\mathbf{B}_{2, q}^{\alpha}(D), \alpha>0,1 \leq q \leq \infty$, if and only if there exists a sequence of vectors $f_{k}=f_{k}(f) \in P W_{a^{k}}(D), a>1, k \in \mathbb{N}$ such that the series $\sum_{k} f_{k}$ converges to $f$ in $\mathcal{H}$ and the following inequalities hold for some $c_{1}>0, c_{2}>0$ which are independent on $f \in \mathbf{B}_{2, q}^{\alpha}(D)$

$$
c_{1}\|f\|_{\mathbf{B}_{2, q}^{\alpha}(D)} \leq\left(\sum_{k=0}^{\infty}\left(a^{k \alpha}\left\|f_{k}\right\|\right)^{q}\right)^{1 / q} \leq c_{2}\|f\|_{\mathbf{B}_{2, q}^{\alpha}(D)}, a>1 .
$$


In the case when $\alpha>0, q=\infty$ one has to make appropriate modifications in the above formulas.

According to (1.4) the functional $\mathcal{E}(f, \omega)$ is a measure of decay of the Spectral Transform $\mathcal{F}_{D}$ and the Theorems 1.1 and 1.2 show that Besov spaces on a manifold $M$ describe decay of the Spectral transform $\mathcal{F}_{D}$ associated with any appropriate operator $D$.

In the case $\mathcal{H}=L_{2}\left(\mathbb{R}^{d}\right)$ the Theorems 1.1 and 1.2 are classical and can be found in [1, [10] and [22]. In the case when $\mathcal{H}$ is $L_{2}$-space on a Riemannian manifold or a graph and $D$ is the square root from the corresponding Laplace operator the Theorems 1 and 2 were proved in our papers [11]-20].

\section{Paley-Wiener subspaces Generated by a SelF-ADJoint operator in a HILBERT SPACE}

Now we describe Paley-Wiener functions for a self-adjoint positive definite operator $D$ in $\mathcal{H}$. According to the spectral theory 3 for any self-adjoint operator $D$ in a Hilbert space $\mathcal{H}$ there exist a direct integral of Hilbert spaces $X=\int X(\lambda) d m(\lambda)$ and a unitary operator $\mathcal{F}_{D}$ from $\mathcal{H}$ onto $X$, which transforms domain of $D^{k}, k \in \mathbb{N}$, onto $X_{k}=\left\{x \in X \mid \lambda^{k} x \in X\right\}$ with norm

$$
\|x(\lambda)\|_{X_{k}}=\left(\int_{0}^{\infty} \lambda^{2 k}\|x(\lambda)\|_{X(\lambda)}^{2} d m(\lambda)\right)^{1 / 2}
$$

besides $\mathcal{F}_{D}\left(D^{k} f\right)=\lambda^{k}\left(\mathcal{F}_{D} f\right)$, if $f$ belongs to the domain of $D^{k}$. As it is known, $X$ is the set of all $m$-measurable functions $\lambda \rightarrow x(\lambda) \in X(\lambda)$, for which the norm

$$
\|x\|_{X}=\left(\int_{0}^{\infty}\|x(\lambda)\|_{X(\lambda)}^{2} d m(\lambda)\right)^{1 / 2}
$$

is finite.

Definition 1. We will say that a vector $f$ from $\mathcal{H}$ belongs to the Paley-Wiener space $P W_{\omega}(D)$ if the support of the Spectral transform $\mathcal{F}_{D} f$ belong to $[0, \omega]$. For a vector $f \in P W_{\omega}(D)$ the notation $\omega_{f}$ will be used for a positive number such that $\left[0, \omega_{f}\right]$ is the smallest interval which contains the support of the Spectral transform $\mathcal{F}_{D} f$.

Using the spectral resolution of identity $P_{\lambda}$ we define the unitary group of operators by the formula

$$
e^{i t D} f=\int_{0}^{\infty} e^{i t \tau} d P_{\tau} f, f \in \mathcal{H}, t \in \mathbb{R} .
$$

Let us introduce the operator

$$
\mathbf{R}_{D}^{\omega} f=\frac{\omega}{\pi^{2}} \sum_{k \in \mathbb{Z}} \frac{(-1)^{k-1}}{(k-1 / 2)^{2}} e^{i\left(\frac{\pi}{\omega}(k-1 / 2)\right) D} f, f \in \mathcal{H}, \omega>0 .
$$

Since $\left\|e^{i t \mathcal{L}} f\right\|=\|f\|$ and

$$
\frac{\omega}{\pi^{2}} \sum_{k \in \mathbb{Z}} \frac{1}{(k-1 / 2)^{2}}=\omega,
$$


the series in (2.2) is convergent and it shows that $\mathbf{R}_{D}^{\omega}$ is a bounded operator in $\mathcal{H}$ with the norm $\omega$ :

$$
\left\|\mathbf{R}_{D}^{\omega} f\right\| \leq \omega\|f\|, f \in \mathcal{H} .
$$

The next theorem contains generalizations of several results from the classical harmonic analysis (in particular the Paley-Wiener theorem) and it follows essentially from our results in 13, 14, 19.

Theorem 2.1. The following statements hold:

(1) the set $\bigcup_{\omega>0} P W_{\omega}(D)$ is dense in $\mathcal{H}$;

(2) the space $P W_{\omega}(D)$ is a linear closed subspace in $\mathcal{H}$;

(3) a function $f \in \mathcal{H}$ belongs to $P W_{\omega}(D)$ if and only if it belongs to the set

$$
\mathcal{D}_{\infty}=\bigcap_{k=1}^{\infty} \mathcal{D}_{k}(D)
$$

and for all $s \in \mathbb{R}_{+}$the following Bernstein inequality takes place

$$
\left\|D^{s} f\right\| \leq \omega^{s}\|f\|
$$

(4) a vector $f \in \mathcal{H}$ belongs to the space $P W_{\omega_{f}}(D), 0<\omega_{f}<\infty$, if and only if $f$ belongs to the set $\mathcal{D}_{\infty}$, the limit

$$
\lim _{k \rightarrow \infty}\left\|D^{k} f\right\|^{1 / k}
$$

exists and

$$
\lim _{k \rightarrow \infty}\left\|D^{k} f\right\|^{1 / k}=\omega_{f}
$$

(5) a vector $f \in \mathcal{H}$ belongs to $P W_{\omega}(D)$ if and only if $f \in \mathcal{D}_{\infty}$ and the upper bound

$$
\sup _{k \in N}\left(\omega^{-k}\left\|D^{k} f\right\|\right)<\infty
$$

is finite,

(6) a vector $f \in \mathcal{H}$ belongs to $P W_{\omega}(D)$ if and only if $f \in \mathcal{D}_{\infty}$ and

$$
\underline{\lim }_{k \rightarrow \infty}\left\|D^{k} f\right\|^{1 / k}=\omega<\infty .
$$

In this case $\omega=\omega_{f}$.

(7) a vector $f \in \mathcal{H}$ belongs to $P W_{\omega}(D)$ if and only if it belongs to the to the set $\mathcal{D}_{\infty}$ and the following Riesz interpolation formula holds

$$
(i D)^{n} f=\left(\boldsymbol{R}_{D}^{\omega}\right)^{n} f, n \in \mathbb{N}
$$

(8) $f \in P W_{\omega}(D)$ if and only if for every $g \in \mathcal{H}$ the scalar-valued function of the real variable $\left\langle e^{i t D} f, g\right\rangle, t \in \mathbb{R}^{1}$, is bounded on the real line and has an extension to the complex plane as an entire function of the exponential type $\omega$

(9) $f \in P W_{\omega}(D)$ if and only if the abstract-valued function $e^{i t D} f$ is bounded on the real line and has an extension to the complex plane as an entire function of the exponential type $\omega$;

(10) $f \in P W_{\omega}(D)$ if and only if the solution $u(t), t \in \mathbb{R}^{1}$ of the Cauchy problem for the corresponding abstract Schrodinger equation

$$
i \frac{\partial u(t)}{\partial t}=D u(t), u(0)=f, i=\sqrt{-1},
$$


has analytic extension $u(z)$ to the complex plane $\mathbb{C}$ as an entire function and satisfies the estimate

$$
\|u(z)\|_{\mathcal{H}} \leq e^{\omega|\Im z|}\|f\|_{\mathcal{H}} .
$$

\section{Direct and Inverse Approximation Theorems}

Now we are going to use the notion of the best approximation (1.2) to introduce Approximation spaces $E_{2, q}^{\alpha}(D), 0<\alpha<r, r \in \mathbb{N}, 1 \leq q \leq \infty$, as spaces for which the following norm is finite

$$
\|f\|_{E_{2, q}^{\alpha}(D)}=\|f\|+\left(\int_{0}^{\infty}\left(s^{\alpha} \mathcal{E}(f, s)\right)^{q} \frac{d s}{s}\right)^{1 / q},
$$

where $0<\alpha<r, 1 \leq q<\infty$, or $0 \leq \alpha \leq r, q=\infty$. It is easy to verify that this norm is equivalent to the following "discrete" norm

$$
\|f\|+\left(\sum_{j \in \mathbb{N}}\left(a^{j \alpha} \mathcal{E}\left(f, a^{j}\right)\right)^{q}\right)^{1 / q}, a>1,
$$

The Plancherel Theorem for $\mathcal{F}_{D}$ also gives the following inequality

$$
\mathcal{E}(f, \omega) \leq \omega^{-k}\left(\int_{\omega}^{\infty}\left\|\mathcal{F}_{D}\left(D^{k} f\right)(\lambda)\right\|_{X(\lambda)}^{2} d m(\lambda)\right)^{1 / 2} \leq \omega^{-k}\left\|D^{k} f\right\| .
$$

In the classical Approximation theory the Direct and Inverse Theorems give equivalence of the Approximation and Besov spaces. Our goal is to extend these results to a more general setting.

For any $f \in \mathcal{H}$ we introduce a difference operator of order $m \in \mathbb{N}$ as

$$
\Delta_{\tau}^{m} f=(-1)^{m+1} \sum_{j=0}^{m}(-1)^{j-1} C_{m}^{j} e^{j \tau(i D)} f, \tau \in \mathbb{R} .
$$

and the modulus of continuity is defined as

$$
\Omega_{m}(f, s)=\sup _{|\tau| \leq s}\left\|\Delta_{\tau}^{m} f\right\|
$$

The following Theorem is a generalization of the classical Direct Approximation Theorem by entire functions of exponential type [10].

Theorem 3.1. There exists a constant $C>0$ such that for all $\omega>0$ and all $f$

$$
\mathcal{E}(f, \omega) \leq \frac{C}{\omega^{k}} \Omega_{m-k}\left(D^{k} f, 1 / \omega\right), 0 \leq k \leq m .
$$

In particular the following embeddings hold true

$$
\mathbf{B}_{2, q}^{\alpha}(D) \subset E_{q}^{\alpha}(D), 1 \leq q \leq \infty .
$$

Proof. If $h \in L_{1}(\mathbb{R})$ is an entire function of exponential type $\omega$ then for any $f \in \mathcal{H}$ the vector

$$
g=\int_{-\infty}^{\infty} h(t) e^{i t D} f d t
$$

belongs to $P W_{\omega}(D)$. Indeed, for every real $\tau$ we have

$$
e^{i \tau D} g=\int_{-\infty}^{\infty} h(t) e^{i(t+\tau) D} f d t=\int_{-\infty}^{\infty} h(t-\tau) e^{i t D} f d t .
$$


Using this formula we can extend the abstract function $e^{i \tau D} g$ to the complex plane as

$$
e^{i z D} g=\int_{-\infty}^{\infty} h(t-z) e^{i t D} f d t
$$

Since by assumption $h \in L_{1}(\mathbb{R})$ is an entire function of exponential type $\omega$ we have

$$
\left\|e^{i z D} g\right\| \leq\|f\| \int_{-\infty}^{\infty}|h(t-z)| d t \leq\|f\| e^{\omega|z|} \int_{-\infty}^{\infty}|h(t)| d t .
$$

It shows that for every functional $g^{*} \in \mathcal{H}$ the function $\left\langle e^{i z D} g, g^{*}\right\rangle$ is an entire function and

$$
\left|\left\langle e^{i z D} g, g^{*}\right\rangle\right| \leq\left\|g^{*}\right\|\|f\| e^{\omega|z|} \int_{-\infty}^{\infty}|h(t)| d t
$$

In other words $\left\langle e^{i z D} g, g^{*}\right\rangle$ is an entire function of the exponential type $\omega$ which is bounded on the real line and application of the classical Bernstein theorem gives the following inequality

$$
\left|\left(\frac{d}{d t}\right)^{k}\left\langle e^{i t D} g, g^{*}\right\rangle\right| \leq \omega^{k} \sup _{t \in \mathbb{R}}\left|\left\langle e^{i t D} g, g^{*}\right\rangle\right| .
$$

Since

$$
\left(\frac{d}{d t}\right)^{k}\left\langle e^{i t D} g, g^{*}\right\rangle=\left\langle e^{i t D}(i D)^{k} g, g^{*}\right\rangle
$$

we obtain for $t=0$

$$
\left|\left\langle D^{k} g, g^{*}\right\rangle\right| \leq \omega^{k}\left\|g^{*}\right\|\|f\| \int_{-\infty}^{\infty}|h(\tau)| d \tau .
$$

Choosing $g^{*}$ such that $\left\|g^{*}\right\|=1$ and $\left\langle D^{k} g, g^{*}\right\rangle=\left\|D^{k} g\right\|$ we obtain the following inequality

$$
\left\|D^{k} g\right\| \leq \omega^{k}\|f\| \int_{-\infty}^{\infty}|h(\tau)| d \tau
$$

which implies that $g$ belongs to $P W_{\omega}(D)$.

Let

$$
h(t)=a\left(\frac{\sin (t / n)}{t}\right)^{n}
$$

where $n \geq m+3$ is an even integer and

$$
a=\left(\int_{-\infty}^{\infty}\left(\frac{\sin (t / n)}{t}\right)^{n} d t\right)^{-1}
$$

With such choice of $a$ and $n$ the function $h$ will have the following properties:

(1) $h$ is an even nonnegative entire function of exponential type one;

(2) $h$ belongs to $L_{1}(\mathbb{R})$ and its $L_{1}(\mathbb{R})$-norm is 1 ;

(3) the integral

$$
\int_{-\infty}^{\infty} h(t)|t|^{m} d t
$$

is finite. 
Consider the following vector

$$
\mathcal{Q}_{h}^{\omega, m}(f)=\int_{-\infty}^{\infty} h(t)\left\{(-1)^{m-1} \Delta_{t / \omega}^{m} f+f\right\} d t,
$$

where

$$
(-1)^{m+1} \Delta_{s}^{m} f=(-1)^{m+1} \sum_{j=0}^{m}(-1)^{j-1} C_{m}^{j} e^{j s(i D)} f=\sum_{j=1}^{m} b_{j} e^{j s(i D)} f-f,
$$

and

$$
b_{1}+b_{2}+\ldots+b_{m}=1 .
$$

The formulas (3.10) and (3.11) imply the following formula

$$
\mathcal{Q}_{h}^{\omega, m}(f)=\int_{-\infty}^{\infty} h(t) \sum_{j=1}^{m} b_{j} e^{j \frac{t}{\omega}(i D)} f d t=\int_{-\infty}^{\infty} \Phi(t) e^{t(i D)} f d t .
$$

where

$$
\Phi(t)=\sum_{j=1}^{m} b_{j}\left(\frac{\omega}{j}\right) h\left(t \frac{\omega}{j}\right) .
$$

Since the function $h(t)$ is of the exponential type one every function $h(t \omega / j)$ is of the type $\omega / j$. It also shows that the function $\Phi(t)$ is of the exponential type $\omega$ as well.

Now we estimate the error of approximation of $f$ by $\mathcal{Q}_{h}^{\omega, m}(f)$. If the modulus of continuity is defined as

$$
\Omega_{m}(f, s)=\sup _{|\tau| \leq s}\left\|\Delta_{\tau}^{m} f\right\|
$$

then since by (3.10)

we obtain

$$
f-\mathcal{Q}_{h}^{\omega, m}(f)=\int_{-\infty}^{\infty} h(t) \Delta_{t / \omega}^{m} f d t
$$

$$
\mathcal{E}(f, \omega) \leq\left\|f-\mathcal{Q}_{h}^{\omega, m}(f)\right\| \leq \int_{-\infty}^{\infty} h(t)\left\|\Delta_{t / \omega}^{m} f\right\| d t \leq \int_{-\infty}^{\infty} h(t) \Omega_{m}(f, t / \omega) d t .
$$

Now we are going to use the following inequalities

$$
\begin{gathered}
\Omega_{m}(f, s) \leq s^{k} \Omega_{m-k}\left(D^{k} f, s\right) \\
\Omega_{m}(f, a s) \leq(1+a)^{m} \Omega_{m}(f, s), a \in \mathbb{R}_{+} .
\end{gathered}
$$

The first one follows from the identity

$$
\Delta_{t}^{k} f=\left(e^{i t D}-I\right)^{k} f=\int_{0}^{t} \ldots \int_{0}^{t} e^{i\left(\tau_{1}+\ldots \tau_{k}\right) D} D^{k} f d \tau_{1} \ldots d \tau_{k},
$$

where $I$ is the identity operator and $k \in \mathbb{N}$. The second one follows from the property

$$
\Omega_{1}\left(f, s_{1}+s_{2}\right) \leq \Omega_{1}\left(f, s_{1}\right)+\Omega_{1}\left(f, s_{2}\right)
$$

which is easy to verify. We can continue our estimation of $E(f, \omega)$.

$$
\mathcal{E}(f, \omega) \leq \int_{-\infty}^{\infty} h(t) \Omega_{m}(f, t / \omega) d t \leq \frac{\Omega_{m-k}\left(D^{k} f, 1 / \omega\right)}{\omega^{k}} \int_{-\infty}^{\infty} h(t)|t|^{k}(1+|t|)^{m-k} d t \leq
$$


where the integral

$$
\frac{C_{m, k}^{h}}{\omega^{k}} \Omega_{m-k}\left(D^{k} f, 1 / \omega\right)
$$

$$
C_{m, k}^{h}=\int_{-\infty}^{\infty} h(t)|t|^{k}(1+|t|)^{m-k} d t
$$

is finite by the choice of $h$. The inequality (3.6) is proved and it implies the second part of the Theorem.

In fact we proved a little bit more. Namely for the same choice of the function $h$ the following holds.

Corollary 3.1. For any $0 \leq k \leq m, k, m \in \mathbb{N}$, here exists a constant $C_{m, k}^{h}$ such that for all $0<\omega<\infty$ and all $f \in \mathcal{H}$ the following inequality holds

$$
\mathcal{E}(f, \omega) \leq\left\|\mathcal{Q}_{h}^{\omega, m}(f)-f\right\| \leq \frac{C_{m, k}^{h}}{\omega^{k}} \Omega_{m-k}\left(D^{k} f, 1 / \omega\right),
$$

where

$$
C_{m, k}^{h}=\int_{-\infty}^{\infty} h(t)|t|^{k}(1+|t|)^{m} d t, 0 \leq k \leq m,
$$

and the operator

is defined in (3.10).

$$
\mathcal{Q}_{h}^{\omega, m}: \mathcal{H} \rightarrow P W_{\omega}(D)
$$

Next, we are going to obtain the Inverse Approximation Theorem in the case $q=\infty$.

Lemma 1. If there exist $r>\alpha-n>0, \alpha>0, r, n \in \mathbb{N}$, such that the quantity

$$
\mathbf{b}_{\infty, n, r}^{\alpha}(f)=\sup _{s>0}\left(s^{n-\alpha} \Omega_{r}\left(D^{n} f, s\right)\right)
$$

is finite, then there exists a constant $A=A(n, r)$ for which

$$
\sup _{s>0} s^{\alpha} \mathcal{E}(f, s) \leq A(n, r) \mathbf{b}_{\infty, n, r}^{\alpha}(f)
$$

Proof. Assume that (3.18) holds, then

$$
\Omega_{r}\left(D^{n} f, s\right) \leq \mathbf{b}_{\infty, n, r}^{\alpha}(f) s^{\alpha-n}
$$

and (3.17) implies

$$
\begin{gathered}
\mathcal{E}(f, s) \leq C_{n+r, n}^{h} s^{-n} \mathbf{b}_{\infty, n, r}^{\alpha}(f) s^{n-\alpha}= \\
A(n, r) \mathbf{b}_{\infty, n, r}^{\alpha}(f) s^{-\alpha} .
\end{gathered}
$$

Lemma is proved.

Lemma 2. If for an $f \in \mathcal{H}$ and for an $\alpha>0$ the following upper bound is finite

$$
\sup _{s>0} s^{\alpha} \mathcal{E}(f, s)=T(f, \alpha)<\infty,
$$

then for every $r>\alpha-n>0, \alpha>0, r, n \in \mathbb{N}$, there exists a constant $C(\alpha, n, r)$ such that the next inequality holds

$$
\mathbf{b}_{\infty, n, r}^{\alpha}(f) \leq C(\alpha, n, r)(\|f\|+T(f, \alpha)) .
$$


Proof. The assumption implies that for a given $f \in \mathcal{H}$ and a sequence of numbers $a^{j}, a>1, j=0,1,2, \ldots$ one can find a sequence $g_{j} \in P W_{a^{j}}(D)$ such that

$$
\left\|f-g_{j}\right\| \leq T(f, \alpha) a^{-j \alpha}, a>1 .
$$

Then for

$$
f_{0}=g_{0}, f_{j}=g_{j}-g_{j-1} \in P W_{a^{j}}(D)
$$

the series

$$
f=f_{0}+f_{1}+f_{2}+\ldots
$$

converges in $\mathcal{H}$. Moreover, we have the following estimates

$$
\left\|f_{0}\right\|=\left\|g_{0}\right\| \leq\left\|g_{0}-f\right\|+\|f\| \leq\|f\|+T(f, \alpha),
$$

$\left\|f_{j}\right\| \leq\left\|f-g_{j}\right\|+\left\|f-g_{j-1}\right\| \leq T(f, \alpha) a^{-j \alpha}+T(f, \alpha) a^{-(j-1) \alpha}=T(f, \alpha)\left(1+a^{\alpha}\right) a^{-j \alpha}$, which imply the following inequality

$$
\left\|f_{j}\right\| \leq C(a, \alpha) a^{-j \alpha}(\|f\|+T(f, \alpha)), j \in \mathbb{N} .
$$

Since $f_{j} \in P W_{a^{j}}(D)$ we have for any $n \in \mathbb{N}$

$$
\left\|D^{n} f_{j}\right\| \leq a^{j n}\left\|f_{j}\right\|, a>1,
$$

we obtain

$$
\left\|D^{n} f_{j}\right\| \leq C(a, \alpha) a^{-j(\alpha-n)}(\|f\|+T(f, \alpha))
$$

which shows that the series

$$
\sum_{j \in \mathbb{N}} D^{n} f_{j}
$$

converges in $\mathcal{H}$ and because the operator $D^{n}$ is closed the sum $f$ of this series belongs to the domain of $D^{n}$ and

$$
D^{n} f=\sum_{j \in \mathbb{N}} D^{n} f_{j} .
$$

Next, let $F_{j}=D^{n} f_{j}$ then we have that $D^{n} f=\sum_{j} F_{j}$, where $F_{j} \in P W_{a^{j}}(D)$ and according to (3.25) and (3.26)

$$
\left\|F_{j}\right\|=\left\|D^{n} f_{j}\right\| \leq a^{j n}\left\|f_{j}\right\| \leq C(a, \alpha) a^{-j(\alpha-n)}(\|f\|+T(f, \alpha)) .
$$

Pick a positive $t$ and a natural $N$ such that

$$
a^{-N} \leq t<a^{-N+1}, a>1,
$$

then we obviously have the following formula for any natural $r$

$$
\Delta_{t}^{r} D^{n} f=\sum_{j=0}^{N-1} \Delta_{t}^{r} F_{j}+\sum_{j=N}^{\infty} \Delta_{t}^{r} F_{j},
$$

where $\Delta_{t}^{r}$ is defined in (3.4). Note, that the Bernstein inequality and the formula (3.16) imply that if $f \in P W_{\omega}(D)$, then

$$
\left\|\Delta_{t}^{r} f\right\| \leq(t \omega)^{r}\|f\| .
$$

Since (3.27) and (3.28) hold we obtain for $j \leq N-1$ the following inequalities

$$
\left\|\Delta_{t}^{r} F_{j}\right\| \leq\left(a^{j} t\right)^{r}\left\|F_{j}\right\| \leq C(a, \alpha)(\|f\|+T(f, \alpha)) a^{j(n+r-\alpha)-(N-1) r)}, a>1 .
$$


These inequalities imply

$$
\begin{gathered}
\left\|\sum_{j=0}^{N-1} \Delta_{t}^{r} F_{j}\right\| \leq C(a, \alpha)(\|f\|+T(f, \alpha)) a^{-r(N-1)} \sum_{j=0}^{N-1} a^{(n+r-\alpha) j}= \\
C(a, \alpha)(\|f\|+T(f, \alpha)) a^{-r(N-1)} \frac{1-a^{(n+r-\alpha) N}}{1-a^{(n+r-\alpha)}} \leq \\
C(a, \alpha, n, r)(\|f\|+T(f, \alpha)) t^{\alpha-n} .
\end{gathered}
$$

By applying the following inequality

$$
\left\|\Delta_{t}^{r} F_{j}\right\| \leq 2^{r}\left\|F_{j}\right\|
$$

to terms with $j \geq N$ we can continue our estimation as

$$
\begin{gathered}
\left\|\sum_{j=N}^{\infty} \Delta_{t}^{r} F_{j}\right\| \leq 2^{r} C(a, \alpha)(\|f\|+T(f, \alpha)) \sum_{j=N}^{\infty} a^{-(\alpha-n) j}= \\
C(a, \alpha) 2^{r}(\|f\|+T(f, \alpha)) a^{-N(\alpha-n)}\left(1-a^{(n-\alpha)}\right)^{-1} \leq \\
C(a, \alpha, n, r)(\|f\|+T(f, \alpha)) t^{\alpha-n} .
\end{gathered}
$$

It gives the following inequality

$$
\left\|\Delta_{t}^{r} D^{n} f\right\| \leq C(a, \alpha, n, r) t^{\alpha-n}(\|f\|+T(f, \alpha)),
$$

from which one has

$$
\Omega_{r}\left(D^{n} f, s\right) \leq C(a, \alpha, n, r)(\|f\|+T(f, \alpha)) s^{\alpha-n}, s>0,
$$

and

$$
\mathbf{b}_{\infty, n, r}^{\alpha}(f) \leq C(a, \alpha, n, r)(\|f\|+T(f, \alpha)) .
$$

The Lemma is proved.

Our main result concerning spaces $\mathbf{B}_{2, \infty}^{\alpha}(D), \alpha>0$, is the following.

Theorem 3.2. The norm of the space $\left.\mathbf{B}_{2, \infty}^{\alpha} D\right), \alpha>0$, is equivalent to the following norms

$$
\begin{gathered}
\|f\|+\sup _{s>0}\left(s^{\alpha} \mathcal{E}(f, s)\right), \\
\left.\|f\|+\sup _{s>0}\left(s^{\alpha} \mathcal{R}(f, s)\right)\right), \\
\|f\|+\sup _{k \in \mathbb{N}}\left(a^{k \alpha} \mathcal{E}\left(f, a^{k}\right)\right), a>1, \\
\left.\|f\|+\sup _{k \in \mathbb{N}}\left(a^{k \alpha} \mathcal{R}\left(f, a^{k}\right)\right)\right), a>1 .
\end{gathered}
$$

Moreover, a vector $f \in \mathcal{H}$ belongs to $\mathbf{B}_{2, \infty}^{\alpha}(D), \alpha>0$, if and only if there exists a sequence of vectors $f_{k}=f_{k}(f) \in P W_{a^{k}}(D), a>1$, such that the series $\sum f_{k}$ converges to $f$ in $\mathcal{H}$ and

$$
c_{1}\|f\|_{\mathbf{B}_{2, \infty}^{\alpha}(D)} \leq \sup _{k \in \mathbb{N}}\left(a^{k \alpha}\left\|f_{k}\right\|\right) \leq c_{2}\|f\|_{\mathbf{B}_{2, \infty}^{\alpha}(D)}, a>1
$$

for certain $c_{1}=c_{1}(D, \alpha), c_{2}=c_{2}(D, \alpha)$ which are independent of $f \in \mathbf{B}_{2, \infty}^{\alpha}(D)$. 
Proof. That the norm of $\mathbf{B}_{2, \infty}^{\alpha}(D), \alpha>0$, is equivalent to any of the norms (3.33)(3.36) follows from the last two Lemmas and (1.4).

Next, if the norm (3.33) is finite then it was shown in the proof of the last Lemma that there exists a sequence of vectors $f_{k}=f_{k}(f) \in P W_{a^{k}}(D), a>1$, such that the series $\sum f_{k}$ converges to $f$ in $\mathcal{H}$. Moreover, the inequality (3.25) shows existence of constant $c$ which is independent of $f \in \mathbf{B}_{2, \infty}^{\alpha}(D)$ for which the following inequality holds

$$
\sup _{k \in \mathbb{N}}\left(a^{k \alpha}\left\|f_{k}\right\|\right) \leq c\|f\|_{\mathbf{B}_{2, \infty}^{\alpha}(D)}, a>1,
$$

Conversely, let us assume that there exists a sequence of vectors $f_{k}=f_{k}(f) \in$ $P W_{a^{k}}(D), a>1$, such that the series $\sum f_{k}$ converges to $f$ in $\mathcal{H}$ and

$$
\sup _{k \in \mathbb{N}}\left(a^{k \alpha}\left\|f_{k}\right\|\right)<\infty \text {. }
$$

We have

or

$$
\begin{gathered}
\mathcal{E}\left(f, a^{N}\right) \leq\left\|f-\sum_{k=0}^{N-1} f_{k}\right\|=\sum_{k=N}^{\infty}\left\|f_{k}\right\| \leq \sup _{k \in \mathbb{N}}\left(a^{k \alpha}\left\|f_{k}\right\|\right) \sum_{k=N}^{\infty} a^{-\alpha j} \leq \\
C \sup _{k \in \mathbb{N}}\left(a^{k \alpha}\left\|f_{k}\right\|\right) a^{-N \alpha},
\end{gathered}
$$

Since we also have

$$
\sup _{N} a^{N \alpha} \mathcal{E}\left(f, a^{N}\right) \leq C \sup _{k \in \mathbb{N}}\left(a^{k \alpha}\left\|f_{k}\right\|\right)
$$

$$
\|f\| \leq \sum_{k}\left\|f_{k}\right\| \leq \sup _{k \in \mathbb{N}}\left(a^{k \alpha}\left\|f_{k}\right\|\right) \sum_{k} a^{-\alpha k}, a>1,
$$

the Theorem is proved.

The Theorems 1.1 and 1.2 from Introduction are extensions of the Theorem 3.2 to all indices $1 \leq q \leq \infty$. Their proofs go essentially along the same lines as the proof of the last Theorem and are omitted.

\section{REFERENCES}

1. J. Akhiezer, Theory of approximation, Ungar, NY, 1956.

2. J. Bergh, J. Lofstrom, Interpolation spaces, Springer-Verlag, 1976.

3. M. Birman and M. Solomyak, Spectral thory of selfadjoint operators in Hilbert space, D.Reidel Publishing Co., Dordrecht, 1987.

4. P. Butzer, H. Berens, Semi-Groups of operators and approximation, Springer, Berlin, 1967.

5. M. Ganzburg, Best constants of harmonic approximation on classes associated with the Laplace operator, J. Approx. Theory 150 (2008), no. 2, 199-213.

6. M. Ganzburg, Limit theorems in spline approximation, J. Math. Anal. Appl. 318 (2006), no. $1,15-31$

7. M. Ganzburg, Limit theorems in approximation theory, Anal. Math. 18 (1992), no. 1, 37-57.

8. S. Krein, I. Pesenson, Interpolation Spaces and Approximation on Lie Groups, The Voronezh State University, Voronezh, 1990,

9. S. Krein, Y. Petunin, E. Semenov, Interpolation of linear operators, Translations of Mathematical Monographs, 54. AMS, Providence, R.I., 1982.

10. S. M. Nikolskii, Approximation of functions of several variables and imbedding theorems, Springer, Berlin, 1975.

11. I. Pesenson, Interpolation spaces on Lie groups, (Russian) Dokl. Akad. Nauk SSSR 246 (1979), no. $6,1298-1303$.

12. I. Pesenson, Nikolskii- Besov spaces connected with representations of Lie groups, (Russian) Dokl. Akad. Nauk SSSR 273 (1983), no. 1, 45-49. 
13. I. Pesenson, The Best Approximation in a Representation Space of a Lie Group, Dokl. Acad. Nauk USSR, v. 302, No 5, pp. 1055-1059, (1988) (Engl. Transl. in Soviet Math. Dokl., v.38, No 2, pp. 384-388, 1989.)

14. I. Pesenson, The Bernstein Inequality in the Space of Representation of Lie group, Dokl. Acad. Nauk USSR 313 (1990), 86-90; English transl. in Soviet Math. Dokl. 42 (1991).

15. I. Pesenson, Approximations in the representation space of a Lie group, (Russian) Izv. Vyssh. Uchebn. Zaved. Mat. 1990, , no. 7, 43-50; translation in Soviet Math. (Iz. VUZ) 34 (1990), no. $7,49-57$

16. I. Pesenson, On the abstract theory of Nikolskii-Besov spaces, (Russian) Izv. Vyssh. Uchebn. Zaved. Mat. 1988, , no. 6, 59-68; translation in Soviet Math. (Iz. VUZ) 32 (1988), no. 6, 80-92

17. I. Pesenson, Bernstein-Nikolskii inequalities and Riesz interpolation formula on compact homogeneous manifolds, J. of Approx. Theory 150, (2008), no. 2, 175-198.

18. I. Pesenson, Paley-Wiener approximations and multiscale approximations in Sobolev and Besov spaces on manifolds, J. Geom. Anal. 19 (2009), no. 2, 390-419.

19. I. Pesenson, A discrete Helgason-Fourier transform for Sobolev and Besov functions on noncompact symmetric spaces, Radon transforms, geometry, and wavelets, 231-247, Contemp. Math., 464, Amer. Math. Soc., Providence, RI, 2008.

20. I. Pesenson, A. Zayed, Paley-Wiener subspace of vectors in a Hilbert space with applications to integral transforms, J. Math. Anal. Appl. 353 (2009), no. 2, 566582.

21. I. Pesenson, M. Pesenson, Sampling, filtering and sparse approximations on graphs, J. of Fourier Analysis and Applications, on line.

22. E. Titchmarsh, Theory of Fourier Integrals, Oxford University Press, 1948.

23. H. Triebel, Theory of function spaces II, Monographs in Mathematics, 84. Birkhuser Verlag, Basel, 1992. 